\title{
Prediction of metal toxicity in aquatic organisms
}

\author{
WANG Wen-Xiong \\ Division of Life Science, The Hong Kong University of Science and Technology, Clearwater Bay, Kowloon, Hong Kong, China
}

Received February 24, 2012; accepted March 30, 2012; published online August 16, 2012

\begin{abstract}
Metal pollution has been a major environmental problem in China with the increasing industrialization. The prediction of metal toxicity is extremely challenging due to the complex metal handling and sequestration strategies of different aquatic organisms. In this review, the recent progress made in this area is discussed. In particular, the subcellular partitioning model which has gained recognition in recent years is highlighted. The subcellular partitioning model appears to be dependable for predicting the toxicity in unicellular phytoplankton. It is important to understand the differential ways that metals bind to different subcellular pools and their ecotoxicological significance in aquatic organisms under different exposure regimes. It is also critical to appreciate that every metal is unique to each aquatic species. Despite the huge progress made over the past 30 years, much remains to be done to fully understand metal toxicity in aquatic organisms.
\end{abstract}

metal pollution, toxicity, aquatic organisms, subcellular distribution, metal sensitive fraction, biologically detoxified metals

Citation: Wang W X. Prediction of metal toxicity in aquatic organisms. Chin Sci Bull, 2013, 58: 194-202, doi: 10.1007/s11434-012-5403-9

Metal pollution in the aquatic environment has been a classical environmental problem in China. As such, numerous studies have been conducted to determine the toxicities of metals to different aquatic organisms over the past several decades. With increasing industrialization in China, incidents of metal pollution in the aquatic environment have risen sharply and are frequently reported by the media [1]. Some of these incidents have seriously affected human health. It is now becoming a significant challenge for both local and central governments to address this classical environmental problem. Based on the policy approved in 2011 for controlling heavy metal pollution, the release of metals $(\mathrm{Pb}, \mathrm{Hg}, \mathrm{Cr}, \mathrm{Cd}$ and $\mathrm{As}$ ) into the environment is expected to be reduced by $15 \%$ by the end of 2015 compared to 2007 levels.

Extensive studies dating back to the early 1980's have been conducted to examine the acute and chronic toxicities of metals to different aquatic organisms [2]. The purposes then were to quantify the lethal concentrations $\left(\mathrm{LC}_{50}\right)$ or

email: wwang@ust.hk effect concentrations $\left(\mathrm{EC}_{50}\right)$ of different metals under different environmental conditions (e.g., temperature, $\mathrm{pH}$, salinity, and others). Much information on the $\mathrm{LC}_{50} / \mathrm{EC}_{50}$ of metals was gathered during this period of study. However, these early studies simply related the exposed ambient concentrations to the toxicological endpoints such as mortality and growth which can be readily measured. Most of the exposed concentrations examined were simply the nominal metal concentrations (spiked concentrations). There were very few studies that directly measured the actual metal concentrations in the exposed medium, not to mention the free metal ion concentrations that are now frequently reported in toxicity studies.

Over the past 30 years, there have been major breakthroughs in studying the metal toxicity in aquatic organisms. Such breakthroughs mainly resulted from the collaboration between chemists and biologists since the chemistry and biology of metals are interrelated. In fact, the interface between biology and chemistry of metals is one of the most fascinating and challenging research areas in environmental science. Some of the major progress made in the past 30 
years in understanding and predicting metal toxicity can be summarized as follows.

First, metal speciation can significantly affect the toxicity of metals $[3,4]$. This has been one of the most active research areas over the past decades. The free ion activity model (FIAM) suggests that the free metal ion is generally the most bioavailable and toxic species [3], although there are many exceptions to this general statement depending on the metals, environmental conditions and organisms. These exceptions are the focus of another longstanding research area, and the influences of metal speciation on metal uptake and toxicity are far from clear. The major contribution of the FIAM is probably more conceptual than practical, in that one really needs to address the chemistry of metals before studying the metal toxicity. Simply reporting the total metal concentrations makes it difficult to generalize the findings to other organisms.

Second, it is now well recognized that metals are bound to many different biological ligands in the organisms $[5,6]$. One example of a biological ligand is the metallothionein (MT), a low molecular weight and cysteine-rich protein with metal homeostasis and detoxification functions. Biological ligands differ in their metal toxicity and sequestration functions, and presumably in their bioavailabilities to higher trophic level. Such complex binding to different biological pools renders it very difficult to directly link the body concentrations and the toxicity together. Thus, an organism with a very high body accumulation may not necessarily be affected by the metals, and an organism with a low body concentration may not necessarily be immune to the metals. In contrast to the many organic contaminants, it is rather difficult to predict the toxicity of metals using the tissue residue approach.

Third, the bioaccumulation of metals in aquatic organisms can now be accurately predicted using the biokinetic modeling approach $[7,8]$. We now have a better understanding of why a particular organism can accumulate a certain amount of metals in their tissues. Similar to the FIAM, the significance of kinetic modeling is probably more conceptual than practical. The model provides a realistic framework to study the bioaccumulation of metals under different conditions. For aquatic animals, there are two basic routes of exposure: aqueous and dietborne. These two routes of exposure were first recognized in the 1970's, but separating them was technically very challenging. Experimental design to separate these two exposure routes is limited by the complicated conditions that the organisms encounter in real environmental settings. With the application of the biokinetic model, a major breakthrough was made over the past decade in recognizing the quantitative importance of dietary exposure (trophic transfer) in most aquatic animals. We now know that in nearly all the major predators (e.g., marine fish and gastropods) and depositfeeding animals, dietary exposure is the predominant route by which the metals are accumulated.
The delineation of metal exposure has significant implication for studying the toxicity of metals [9]. The traditional approach of conducting toxicity testing with only aqueous exposure may thus be unable to reflect the real situation in the natural environments where aquatic animals accumulate metals mostly through trophic transfer (dietary route). Water quality criteria established based on waterborne metal tests may not be able to protect aquatic organisms. Dietary exposure and its implications will continue to be a major research focus in toxicity studies in the future.

In this review, I will mainly address the prediction of metal toxicity in aquatic organisms. It is not the purpose of this review to summarize all the toxicity tests of different metals in different aquatic organisms. Instead, I will review approaches to predicting metal toxicity, in particular the subcellular approach being developed over the past few years [10].

\section{The free ion activity model and the biotic ligand model}

Several approaches are now available to predict the toxicity of metals to aquatic organisms, including the free ion activity model (FIAM), the biotic ligand model (BLM), and the subcellular partitioning model (SPM) (Figure 1). The FIAM was first developed in the 1970's and was reviewed by Campbell [11]. Strictly speaking, the FIAM is used only to predict the uptake/bioavailability of metals instead of the toxicity since it only deals with the transport of metals across the biological membranes. The applicability of FIAM has been tested in many organisms under different conditions. The model can now be considered a conceptual model to explain the relationship between metal speciation and metal bioavailability/toxicity. Two main processes are considered in the FIAM: the kinetic exchange on the cell surface of the free ion metal species and the internalization. In essence, FIAM is a chemical equilibrium model and does not consider the complicated handling/sequestration of metals

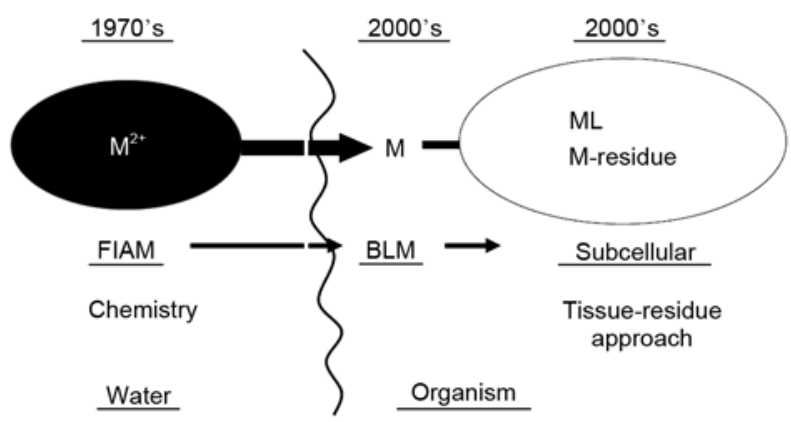

Figure 1 Development of different models to study metal toxicity in aquatic organisms. The free ion activity model (FIAM) was proposed in the 1970's, while the biotic ligand model (BLM) and subcellular partitioning model were proposed in the 2000 's. $\mathrm{M}^{2+}$ : free metal ion; M: metal; ML: metal binding ligand. 
inside the organisms.

The BLM was first proposed by Di Toro et al. [12]. Since its first publication, their paper has been cited over 420 times, highlighting the rapid development of the model and its applications to predict the toxicity of metals in aquatic organisms. This model is essentially based on the FIAM but provides a better understanding of metal chemistry and physiological effects (Figure 2). Most of the earlier studies were based on the fish gills, considered to represent the biotic ligands, with some further studies on small organisms such as phytoplankton and daphnids. One of the main drivers for the development of the BLM was the very extensive studies of the binding of metals to the fish gills (especially the rainbow trout as the model species), including the binding density and stability constant which are affected by competing ions and other physicochemical parameters. Fish gills are generally sensitive to metal toxicity and are the main sites for different physiological functions such as acid-base balance, gas exchanges, ion pumps and waste excretion. In this regard, it is reasonable to select fish gills as the sensitive organs in developing the BLM. Therefore, one of the most important assumptions for the BLM is that the biotic ligand must be sensitive to metal toxicity.

The BLM can be considered an extension of the FIAM since it is used to predict the degree of metal binding at the site of action and this level of accumulation is in turn related to a toxicological response [13]. Both models are based on several assumptions and a number of exceptions have been documented $[14,15]$. Similar to the FIAM, the BLM is an equilibrium model, but the main contribution of this model is that it incorporates basically all the main frameworks of ecotoxicology, namely speciation (chemistry), bioaccumulation (bioavailability) and toxicity. The framework of the BLM is shown in Figure 2. With the known toxic metal concentrations in the biotic ligands (e.g., fish gills) and the binding stability constant, the model calculates the likely toxic metal free ion concentrations in the environments, and the likely toxic metal concentrations by considering metal complexation (dissolved organic matter)

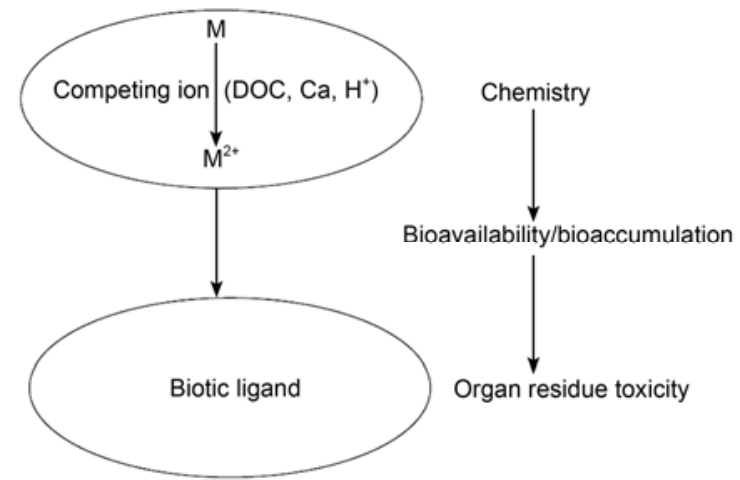

Figure 2 A schematic illustration of the biotic ligand model (left) showing its consistence with the ecotoxicological study framework (right). $\mathrm{M}^{2+}$ : free metal ion; M: metal. and competition (e.g., $\mathrm{Ca}, \mathrm{Mg}, \mathrm{H}^{+}$).

As expected, development of the BLM would require numerous parameters that are site specific, such as the relationship between toxicity and biotic ligand metal concentration, the binding stability constants of metals and the biotic ligands, and the chemical compositions of the medium. Given such major requirements for the development of the model, it is applicable to only a few metals at present (such as $\mathrm{Cu}, \mathrm{Ag}, \mathrm{Zn}, \mathrm{Ni}$ ) and a few model species such as fish, phytoplankton and daphnids. More studies are needed for other metals and other aquatic species. It will be a challenge to develop such a model in large aquatic species in which metal biology is much more complicated (as will be detailed later). Selecting the "right" sensitive biotic ligand will also be a major challenge since the site of toxic action is not simply the fish gills. Gills in marine fish are not the only site of metal uptake; there are other available sites for metal entry into the marine fish (e.g., gastrointestinal metal uptake). In addition, the variability of the binding stability constant still needs to be verified under different conditions; this will be an important area of further study.

Most of the applications of the BLM are on acute toxicities of metals, with few addressing the metal chronic toxicity. This is somewhat understandable since the binding of metals during chronic exposure may be fundamentally different from that under acute exposure. In this regard, it is critical to study how metals are distributed in the organisms during chronic exposure. In addition, the application of the BLM to dietary exposure is still in its infancy.

\section{The subcellular partitioning model}

It is well known that metals are bound to different subcellular fractions, each with its own biological significance. The metal handling and sequestration strategies of different aquatic organisms are extremely complex. Both the FIAM and the BLM are chemical equilibrium-based and neither considers the internal metal fractionation (or handling). Over the past decade, with the application of differential centrifugation and physical and chemical treatments, it is possible to fractionate the cellular metals into five different fractions: the cellular debris, organelles, metal-rich granules (MRG), heat-denatured proteins (HDP), and heat-stable proteins (HSP) [16-20]. These methods are now routinely used to study the distribution of metals in different subcellular fractions, and the studies have demonstrated the importance of these fractions in trophic transfer. About 10 years ago, Wallace et al. [20] further proposed the metal sensitive fraction (MSF) which combines organelles and HDP, and the biologically detoxified metal fraction (BDM) which combines MRG and HSP. The MSF is considered the target of attack of metals in cells, while the BDM is considered to alleviate toxicity. In another paper, Wallace and Luoma [21] proposed the trophically bioavailable metal 
(TAM) fraction which combines organelles, HDP and HSP. Over the past few years, many studies have been conducted to examine the usefulness of the TAM in predicting the trophic transfer of metals, and the results are often more cautious than certain [22]. For example, the definition of the TAM excludes the cellular debris and the MRG fractions, but studies using purified fractions have demonstrated that metals in these two fractions are indeed also bioavailable to predators [23,24]. However, it does make ecotoxicological sense to consider MRG and HSP as the BDM, and to consider HDP and organelles as the MSF.

A large dataset is now available on the subcellular distribution of metals in different groups of aquatic organisms under different environmental conditions. In recent years, several studies have attempted to address whether the subcellular partitioning can provide a reasonable prediction of metal toxicity. Wang and Rainbow [10] termed it the subcellular partitioning model (SPM) (Figure 3). This approach can be considered an extension of the tissue residue approach as it considers the critical sites of metal toxic actions. In other words, it combines the total tissue residue concentration and the subcellular fraction to predict the toxicity of metals. The premise of the SPM is that the total concentration of metals in the biological tissues cannot predict metal toxicity given the complexity of metal binding. This is somewhat in contrast to the organic contaminants since the lipid soluble fraction may be the major toxic action of these compounds.

\subsection{Phytoplankton}

Thus far, tests using the SPM have mostly focused on small marine phytoplankton and freshwater cladocerans. Miao and Wang [25] tested the accumulation and internal distribution of $\mathrm{Cd}$ in a marine diatom Thalassiosira weissflogii and dinoflagellate Prorocentrum minimum. They divided the cellular metals into soluble and insoluble metals, which were separated by ultra-centrifugation of the cellular

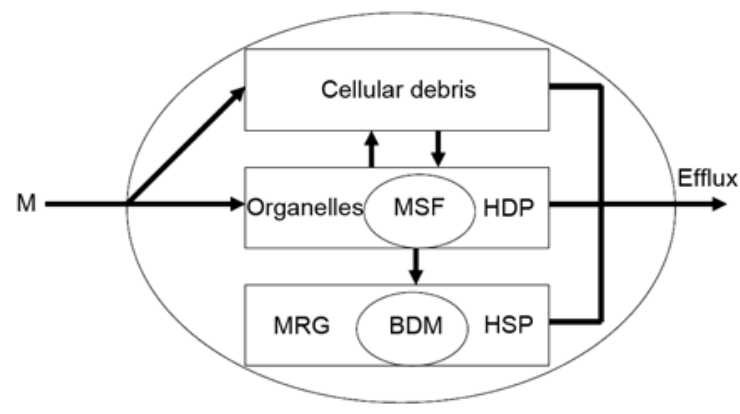

Figure 3 A schematic illustration of the subcellular partitioning model. M: metal; HDP: heat-denatured proteins; HSP: heat-stable proteins; MRG: metal-rich granules; MSF: metal-sensitive fraction; BDM: biologically detoxified fraction. Note that the figure assumes that metals first interact with the cellular debris and metal sensitive fraction, and efflux of metals is from all subcellular fractions. homogenates at $235 \mathrm{~kg}$ for $30 \mathrm{~min}$. They found that among the different types of $\mathrm{Cd}$ concentrations, the soluble $\mathrm{Cd}$ was the best predictor (among intracellular $\mathrm{Cd}$, adsorbed $\mathrm{Cd}$, and $\left[\mathrm{Cd}^{2+}\right]$ ) of $\mathrm{Cd}$ inhibition of algal growth and photosynthesis under different nutrient conditions. In this study, the five subcellular fractions were not separated, but the insoluble fraction was probably a combination of MRG, cellular debris and organelles, and the soluble fraction was probably a combination of HDP and HSP. In the diatom T. weissflogii, Cd was mostly distributed in the insoluble fraction, whereas in the dinoflagellate $P$. minimum, $\mathrm{Cd}$ was more evenly distributed between the insoluble and soluble fractions. The difference in toxicity among cells treated with different nutrients was the smallest when the $\mathrm{Cd}$ concentration in the soluble fraction was used, suggesting that it may be the best predictor of $\mathrm{Cd}$ toxicity under different nutrient conditions. In another study, Miao and Wang [26] examined the accumulation and subcellular distribution of $\mathrm{Cu}$ in the same diatom T. weissflogii under different nutrient conditions ( $\mathrm{N}$ and $\mathrm{P}$ enrichment and starvation, and different $\mathrm{N}$ sources). Among the different $\mathrm{Cu}$ concentration parameters examined (intracellular $\mathrm{Cu}$, adsorbed $\mathrm{Cu}$, and $\left[\mathrm{Cu}^{2+}\right]$, and subcellular $\mathrm{Cu}$ ), the intra- $\mathrm{Cu}$ was the best $\mathrm{Cu}$ toxicity predictor since it accounted for most of the $\mathrm{Cu}$-induced response between cells treated with different nutrients. For example, there was a $142 \times$ difference between the highest and lowest $\left[\mathrm{Cu}^{2+}\right]-$ $\mathrm{IC}_{50}$ (median inhibition concentration), but only a $3.9 \times$ difference between the highest and lowest intra- $\mathrm{IC}_{50}$, in diatoms treated with different nutrients. The study provided new insights into predicting $\mathrm{Cu}$ toxicity using the metal intracellular concentration and its subcellular distribution.

In subsequent experiments, the distribution of metals in the phytoplankton was further divided into the five subcellular fractions described above instead of just the soluble and insoluble fractions $[27,28]$. In a series of studies, Wang and Wang [27,28] explored whether patterns in cellular or subcellular $\mathrm{Cd}$ distributions could be a good predictor of $\mathrm{Cd}$ toxicity in marine diatoms Thalassiosira nordenskioeldii, under different light irradiance and temperature conditions. In these studies, they separated the $\mathrm{Cd}$ in the diatoms into the five biologically relevant fractions (MRG, cellular debris, organelles, HDP and HSP) and also considered the partitioning of $\mathrm{Cd}$ to two subcellular compartments comprising MSF and BDM. In this diatom, Cd was mostly bound to HSP, and was redistributed with increasing $\left[\mathrm{Cd}^{2+}\right]$ concentration from the BDM to presumably the MSF, leading to higher cellular $\mathrm{Cd}$ accumulation, toxicity and sensitivity. Interestingly, the calculated $\mathrm{IC}_{50}$ based on MSF or organelles exhibited the smallest variance among the different irradiance treatments, suggesting that MSF or organelles may be the best predictor of Cd toxicity under different irradiance levels (Figure 4). In contrast, the calculated $\mathrm{IC}_{50} \mathrm{~S}$ of $\left[\mathrm{Cd}^{2+}\right]$ differed by $3.9 \times$ among the different irradiance treatments, thus differences in Cd toxicity cannot be entirely explained by $\left[\mathrm{Cd}^{2+}\right]$ because the diatoms displayed 

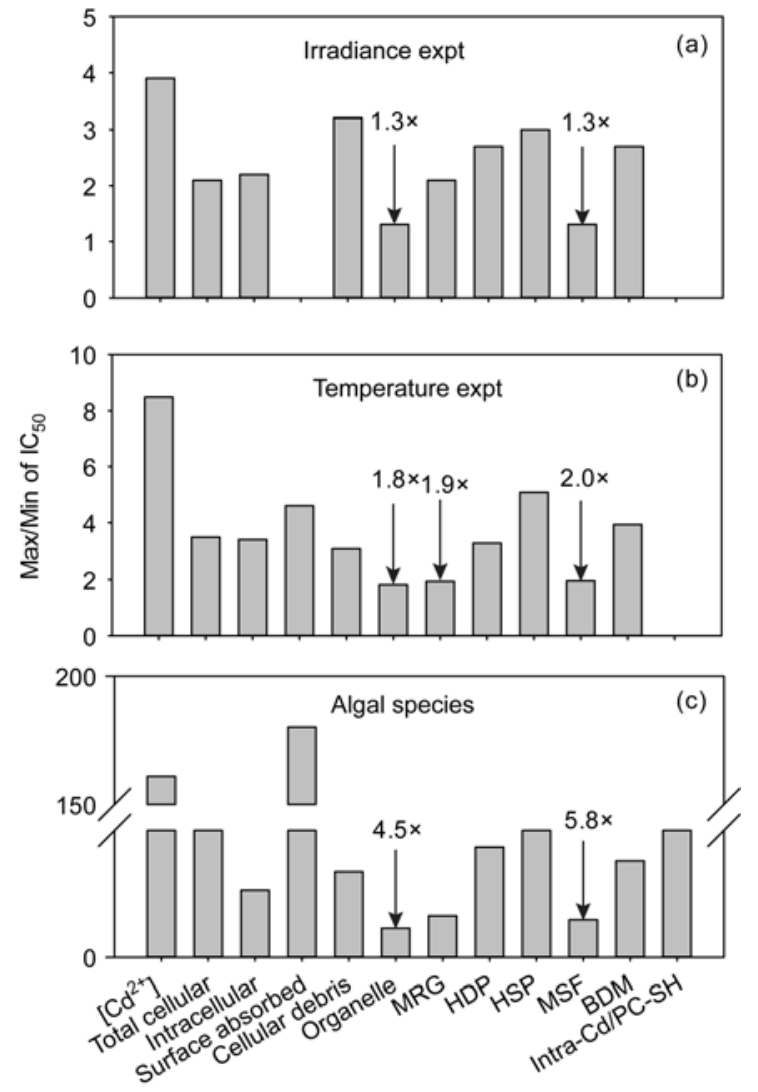

Figure 4 The difference between the maximum and minimum median inhibition concentrations $\left(\mathrm{IC}_{50}\right)$ of the cellular growth rate of diatom Thalassiosira nordenskioeldii based on the $\mathrm{Cd}$ concentration in the water $\left[\mathrm{Cd}^{2+}\right]$ or in different cellular fractions at different irradiances (a) and temperatures (b), and for different species of phytoplankton (c). Data modified from Wang and Wang [27,28,30]. MRG: metal-rich granules; HDP: heat-denatured protein; HSP: heat stable protein; MSF: metal sensitive fraction; BDM: biologically detoxified metals.

different bioaccumulation potentials. In this study, there was similarly a strong correlation between the cellular accumulation (surface adsorbed-Cd, total cellular $\mathrm{Cd}$ and intracellular $\mathrm{Cd}$ ) and the growth rate inhibition, and the BLM may be reasonably used to predict $\mathrm{Cd}$ toxicity in marine diatoms. This study provided the first experimental evidence that the MSF can predict Cd toxicity in marine phytoplankton.

Wang and Wang [28] then used a similar approach to test Cd toxicity in the same diatom $T$. nordenskioeldii under different temperatures, coupled with the measurements of thiol induction. In their study, a higher temperature increased the $\mathrm{Cd}$ toxicity due to increased cellular $\mathrm{Cd}$ accumulation or a poorer detoxification ability. Again, Cd concentration in MSF or organelles served as the best indicator of $\mathrm{Cd}$ toxicity in diatoms at different temperatures since the difference among the different temperature treatments was the smallest among the different $\mathrm{Cd}$-concentration based indicators (Figure 4). The organelle $\mathrm{Cd}$ (org-Cd) based $\mathrm{IC}_{50}$ and MSF-based Cd-IC 50 values were also directly comparable with those determined under different irradiances [27] quantified under the same conditions, which suggested the high reproducibility of these values.

It has been well recognized that phytoplankton synthesize phytochelatins (PCs) with a common structure of $(\gamma \text {-EC })_{n}$-Gly $\left(\mathrm{PC}_{n}, n=2-11\right)$ under metal stress conditions [29]. PCs are rich in cysteine and chelate metals through thiol groups in their amino acids, playing an important role in metal detoxification and tolerance in plants. Wang and Wang [28] also found that PCs were induced by $\left[\mathrm{Cd}^{2+}\right]$ and $\mathrm{PC}-\mathrm{SH}\left(2 \times \mathrm{PC}_{2}+3 \times \mathrm{PC}_{3}+4 \times \mathrm{PC}_{4}\right)$ is a biomarker for cellular $\mathrm{Cd}$ stress given the significant relationship between the intracellular Cd and PC-SH concentration. Nevertheless, there may be additional detoxification mechanisms for the diatoms since the intracellular $\mathrm{Cd} / \mathrm{PC}-\mathrm{SH}$ ratio did not always explain the temperature-dependent $\mathrm{Cd}$ tolerance.

In another study, Wang and Wang [30] attempted to explain the differential $\mathrm{Cd}$ sensitivity in three marine phytoplankton (the diatom Thalassiosira pseudonana, the dinoflagellate Prorocentrum minimum and the green alga Chlorella autotrophica), again, based on $\mathrm{Cd}$ accumulation, subcellular distribution, and PCs synthesis. Among these three species, the diatom $T$. pseudonana was the most sensitive, and $C$. autotrophica was the most tolerant, while the dinoflagellate $P$. minimum was in-between. Subcellular distribution appeared to explain to some extent the difference in $\mathrm{Cd}$ tolerance among the three phytoplankton species. For example, C. autotrophica had the highest percentage of cellular debris-Cd, which helped to reduce the amount of intra-Cd ending up in the MSF. Indeed, this green alga had the lowest org-Cd concentration, whereas the diatom $T$. pseudonana had the highest org- $\mathrm{Cd}$, corresponding to their relative tolerance to $\mathrm{Cd}$. T. pseudonana had the lowest percentage of $\mathrm{Cd}$ in the cellular debris fraction. $\mathrm{The} \mathrm{IC}_{50}$ values calculated from org-Cd showed the least variation compared with other subcellular pools (Figure 4). These results strongly indicated that org-Cd may explain the interspecies difference in Cd sensitivity, similar to earlier studies conducted under different temperature and irradiance conditions [27,28]. In this study, the MSF-Cd appeared to be less useful in explaining the interspecies differences in Cd sensitivity. One of the main reasons was that HDP-Cd accounted for as high as 58\% of MSF-Cd in C. autotrophica and $41 \%$ of MSF-Cd in P. minimum, leading to the different patterns of MSF-Cd and org-Cd accumulation. In previous studies $[27,28]$, only $<13 \%$ of total MSF-Cd was attributed to HDP-Cd in the diatoms. It is possible that the toxicity action sites (e.g., photosystem) of Cd may differ among algal species.

In addition to the organelle fraction, the BDM may also explain the interspecies difference in Cd toxicity in phytoplankton. For example, C. autotrophica had a high percentage of HSP-Cd at a low $\left[\mathrm{Cd}^{2+}\right]$, suggesting the high capacity of HSP to bind to Cd with a correspondingly high tolerance. However, the involvement of HSP in detoxification was not obvious in the other two species, especially $P$. minimum. At 
a high $\left[\mathrm{Cd}^{2+}\right]$, instead of spilling into the MSF, there was a large increase in the MRG fraction in C. autotrophica, indicating that $\mathrm{Cd}$ was probably channeled to MRG for detoxification. Metal detoxification strategies may therefore vary between HSP and MRG under different metal stress conditions. The interplay between HSP and MRG can offer an excellent opportunity to study metal handling and detoxification in marine phytoplankton.

Wang and Wang [30] also found that the intra-Cd/PC-SH stoichiometry, instead of simply PC induction, could explain the differing sensitivities. For example, the diatom had much higher PC synthesis than the other two species, but it also had the highest sensitivity to $\mathrm{Cd}$ exposure. The PC detoxification appears to be the lowest in this diatom given its lowest intra-Cd/PC-SH toxicity threshold. There was a positive relationship between intra-Cd/PC-SH and growth inhibition in all three species, but the importance of PCs to the whole detoxification system differs among species. Interestingly, when the intra-Cd/PC-SH reached 33, the growth inhibition in $C$. autotrophica suddenly slowed down the per unit increase of the intra- $\mathrm{Cd} / \mathrm{PC}-\mathrm{SH}$ ratio. There may be other mechanisms for $\mathrm{Cd}$ detoxification to alleviate the growth inhibition in $C$. autotrophica.

More recently, $\mathrm{Wu}$ and Wang [31] also examined the interspecies difference in $\mathrm{Hg}(\mathrm{II})$ and $\mathrm{MeHg}$ sensitivities among three marine phytoplankton (the diatom $T$. pseudonana, the green alga $C$. autotrophica, and the flagellate Isochrysis galbana), coupled with accumulation and subcellular distribution measurements. For $\mathrm{MeHg}$, the interspecies toxic difference varied by a factor of 1.80 , with $C$. autotrophica being the most sensitive to $\mathrm{MeHg}$ and T. pseudonana the least sensitive. Difference among the three species was the least when the $\mathrm{MeHg}$ concentration was expressed by organelles, MSF, or MRG (Figure 5), again demonstrating that these subcellular fractions were able to explain the interspecies difference in $\mathrm{MeHg}$ sensitivity. The diatom T. pseudonana had the lowest cellular burden while C. autotrophica had the highest, consistent with their respective sensitivities towards $\mathrm{MeHg}$ exposure.

Data for $\mathrm{Hg}(\mathrm{II})$ were rather in contrast to those for $\mathrm{MeHg}$. C. autotrophica, which accumulated the lowest level of $\mathrm{Hg}(\mathrm{II})$, was the most tolerant as expected. T. pseudonana accumulated much higher levels of total and intracellular $\mathrm{Hg}$ (II) than I. galbana did even though they showed similar sensitivities. One of the explanations was that $T$. pseudonana had a higher percentage of $\mathrm{Hg}$ (II) in the cellular debris than did I. galbana, implying that the cellular debris may play an important role in $\mathrm{Hg}$ (II) detoxification. Among the three species, the $\mathrm{IC}_{50}$ values calculated from the concentrations of $\mathrm{Hg}$ (II) in the BDM and the HSP showed the smallest variations $(2.0 \times$ and $1.6 \times$, respectively), indicating that these indexes may be able to explain the difference in interspecies sensitivity (Figure 5). These $\mathrm{Hg}$ (II) data are somewhat different from those of Wang and Wang [30] demonstrating that MSF or organelles was the best predictor

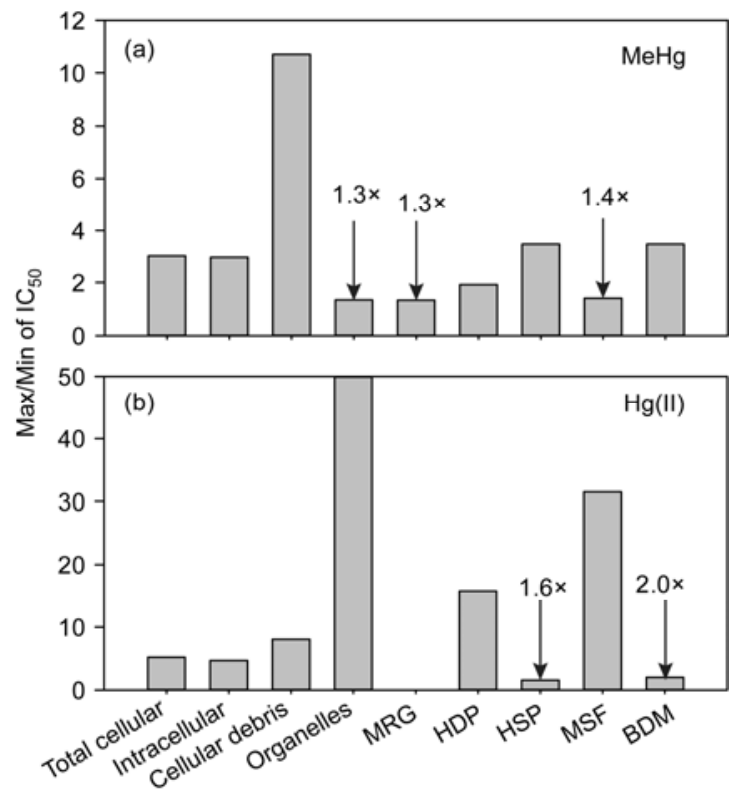

Figure 5 The difference between the maximum and minimum median inhibition concentrations $\left(\mathrm{IC}_{50}\right)$ of the cellular growth rate of three species of marine phytoplankton for $\mathrm{MeHg}$ (a) and $\mathrm{Hg}$ (II) (b). Data modified from $\mathrm{Wu}$ and Wang [31]. MRG: metal-rich granules; HDP: heat-denatured protein; HSP: heat stable protein; MSF: metal-sensitive fraction. BDM: biologically detoxified metals.

of the growth inhibition of marine algae under Cd stress. Detoxification was probably the driving reason for the differential sensitivities of different phytoplankton species to $\mathrm{Hg}(\mathrm{II})$.

\subsection{Invertebrates}

In the animal system, there are few applications of the SPM in predicting toxicity. The toxicological implications of different pools of metals remain rather poorly explored. Earlier, Rainbow [6] hypothesized that when accumulated metal destined for storage in detoxified form (e.g., by MT or MRG) exceeds the detoxified binding capacity, the metals are subsequently bound to other (metabolically available) fractions, with the potential to cause toxicity to the organisms. Wallace et al. [32] demonstrated that the prey capture efficiency of the grass shrimp Palaemonetes pugio was correlated with $\mathrm{Cd}$ concentrations in the high molecular weight proteins (presumably the HDP). In several studies [33,34], the relationships between $\mathrm{Cd}$ accumulation and internal distribution, and growth and mortality were examined in a freshwater bivalve Pyganodon grandis in transplant experiments. Bivalves transplanted to the most contaminated sites contained the highest $\mathrm{Cd}$ concentrations in the high molecular weight pool (HMW>25 kDa) as well as in the metallothionein pool (Cd-MT). These data suggested that excessive accumulation of $\mathrm{Cd}$ in the HMW pool of the gill cytosol of the bivalves may be related to the impairment of population health. However, these authors also noted the difficulty of assigning any 
predictive role to internal metal partitioning. In these studies, $\mathrm{Cd}$ was partitioned into a proposed metal- sensitive fraction consisting of HSP and organelles and a metal detoxified fraction consisting of MT and granules. The distribution of $\mathrm{Cd}$ in these fractions is dependent on metal concentration, season, species, and route of exposure [35-37].

Tsui and Wang [38] used the internal distribution approach to predict the variations of acute $\mathrm{Hg}$ toxicity in Daphnia magna under different temperatures, population origins, body sizes, and $\mathrm{Hg}$ pre-exposure. Detoxification and increased tolerance of daphnids to $\mathrm{Hg}$ can be achieved by one of mechanisms such as reduced $\mathrm{Hg}$ (II) uptake, increased intrinsic tolerance, and increased detoxification. Correlating $\mathrm{Hg}$ levels in different compartments with the daphnid survival rate resulted in the following sequence: aqueous $\mathrm{Hg}>$ whole body $\mathrm{Hg}>\mathrm{Hg}$ in the MSF. However, the threshold lethal concentration of $\mathrm{Hg}$ (concentration causing $1 \%$ mortality) based on the concentration of $\mathrm{Hg}$ in the MSF was the best indicator of $\mathrm{Hg}$ toxicity. Therefore, the fractionation approach is more useful for explaining sub-lethal $\mathrm{Hg}$ toxicity than acute toxicity. This study was probably the first to use the subcellular fraction approach to study the metal acute toxicity in daphnids.

In a subsequent study, Wang and Guan [39] examined the subcellular partitioning of $\mathrm{Zn}$ in D. magna under both acute and chronic exposure, again with an aim to test whether metal associated with a specific subcellular fraction can serve as a better predictor of metal toxicity. When the daphnids were exposed to different $\mathrm{Zn}$ concentrations for 48 $\mathrm{h}$ or to one lethal concentration $(1000 \mu \mathrm{g} / \mathrm{L})$ for different durations (time-to-death for up to $47 \mathrm{~h}$ ), mortality occurred when the newly accumulated $\mathrm{Zn}$ concentration reached a threshold level of $40 \mu \mathrm{g} / \mathrm{g}$ wet weight (or $320 \mu \mathrm{g} / \mathrm{g}$ dry weight). Interestingly, the window for acute $\mathrm{Zn}$ toxicity appeared to be extremely narrow, which strongly suggested that there was a threshold level at which acute Zn toxicity was triggered. Thus, it was difficult to observe a typical tissue-dose-versus-toxicity relationship for $\mathrm{Zn}$ in Daphnia. For example, at 250 to $340 \mu \mathrm{g}(\mathrm{Zn}) / \mathrm{L}$, the mortality rate varied over a range of $18 \%$ to $93 \%$ as the body $\mathrm{Zn}$ concentrations varied only between 200 to $281 \mu \mathrm{g} / \mathrm{g}$ dry weight. In these acute toxicity tests, $\mathrm{Zn}$ was channelled mostly into the cellular debris fraction, which was presumably the first targeted site of $\mathrm{Zn}$ binding. This study also showed that the MSF did not predict the acute Zn toxicity in Daphnia, but a tissue-based $\mathrm{Zn}$ accumulation approach with a threshold $\mathrm{Zn}$ tissue concentration $(40 \mu \mathrm{g} / \mathrm{g}$ wet weight) was much better at it.

The lethal threshold concentration of $400 \mu \mathrm{g} / \mathrm{g}$ dry weight for $\mathrm{Zn}$ in daphnids during acute toxicity tests has a major implication for metal toxicity prediction in daphnids. It will be difficult to calculate the $50 \%$ lethal tissue concentration $\left(\mathrm{LT}_{50}\right)$ simply based on the dose-response relationship. In the BLM, an inherent assumption is that the $\mathrm{LT}_{50}$ can be calculated from $\mathrm{LC}_{50}$. However, the relationship between the total $\mathrm{Zn}$ accumulation and mortality was not sigmoid as classically depicted. Mortality coincided with the saturation of $\mathrm{Zn}$ accumulation, but no obvious lethality occurred below saturation. It would therefore be interesting to specifically test the lethal $\mathrm{Zn}$ concentration under different physicochemical conditions to further improve the BLM.

One of the most interesting ecotoxicological aspects regarding cladocerans is that there are clear differences in metal toxicity among different species. Shaw et al. [40] showed that D. magna was the most tolerant species to $\mathrm{Zn}$ and $\mathrm{Cd}$ among the different cladocerans species they examined (Daphnia ambigua, Ceriodaphnia dubia, Daphnia pulex, and D. magna). The concentration-response relationships for $\mathrm{Cd}$ and $\mathrm{Zn}$ in D. magna were also very different from the other daphnid species. It is still not clear whether such differences may be caused by the subcellular partitioning of metals, as is the case for marine phytoplankton discussed above.

During chronic exposure (14 d), there was no observable effect on survivorship and growth at a newly accumulated tissue concentration of over $40 \mu \mathrm{g} / \mathrm{g}$ wet weight. Thus, the critical body tissue concentration for $\mathrm{Zn}$ became higher during chronic exposure than it did during acute challenge. This increased tolerance may have been due to the acclimation of daphnids to $\mathrm{Zn}$ or induction of $\mathrm{Zn}$ detoxification. $\mathrm{Zn}$ was mainly found in the organelles and HSP fractions during chronic exposure, and subcellular fractionation also could not predict the chronic toxicity of $\mathrm{Zn}$ in Daphnia.

\subsection{Metabolically available metals}

There is a rather complex relationship between toxicity and total accumulated metal concentration in most invertebrates capable of detoxification through storing away the accumulated metals. Metals are stored in different forms inside the organisms. One school of thought is that metals in the animals can be divided into different forms: detoxified metals (including storage) and metabolically available metals, or the metal active pool and the metal inactive pool. In a recent study, Rainbow and Luoma [41] constructed a biodynamic model of $\mathrm{Zn}$ bioaccumulation in metabolically available and detoxified components in three crustaceans: the caridean decapod Palaemon elegans, the amphipod Orchestia gammarellus and the barnacle Amphibalanus amphitrite. The bioaccumulation model was then linked to the onset of toxic $\mathrm{Zn}$ effects in the crustaceans. The premise is that toxicity is related to the total $\mathrm{Zn}$ flux rate and the metabolically available $\mathrm{Zn}$ concentration instead of the total accumulated metal concentration. Rainbow and Luoma [41] argued that a threshold critical accumulated body concentration cannot account for the metal toxicity, except under specific circumstances in which there was a lack of storage detoxification. Instead, there is a threshold concentration for the metabolically available metal, which may be used to explain the acute toxicity of metals. The ratio of the threshold concen- 
tration of metabolically available metal to the total accumulated body concentration will vary for different species and under different conditions. Toxicity is induced if the metabolically available concentration exceeds the toxic threshold concentration, or if the $\mathrm{Zn}$ influx rate exceeds the combined rates of detoxification and excretion.

The definition of metabolically available metal is somewhat conceptual, unlike the definition of subcellular pools, which are of course operationally defined as well. Rainbow and Luoma [41] defined metabolically available metal as the metal available to bind in the "correct" place to play an essential role, and presumably metal that is already bound "correctly" to play such a role, as in metallo-enzymes. Thus, the metabolically available metal may include firstly the metallothionein (MT), and then the organelles and HSP. Rainbow and Luoma [41] suggested that the metabolically available metal may be estimated theoretically from the essential metabolic requirements of metabolizing tissue, or by measuring the minimum metal concentrations in tissues.

It has been assumed that newly accumulated metals will first enter metabolically available pools, followed by excretion or detoxification. Several studies have tested this hypothesis in marine bivalves [42,43]. Pan and Wang [43] conducted a series of short-term experiments on the dynamics of subcellular distribution of $\mathrm{Cd}$ and $\mathrm{Zn}$ in the scallop Chlamys nobilis following dissolved and dietary metal challenges. Significant changes were found in the scallops when they were challenged with different concentrations of metals in the aqueous and food phases. The main binding pool for the newly acquired metals was organelles for $\mathrm{Cd}$ and the cellular debris for Zn. HSP was the most important storage pool for $\mathrm{Cd}$ in the scallops. In the green mussel Perna viridis, the HDP (involving enzymes) and presumably cellular debris were the first ligands to bind to the metals during the dissolved exposure [42]. These metals were then redistributed from the HDP to HSP, assuming that the first targets of metal attack are the metabolically available pools, and subsequently excreted or stored. However, it is clear that different metals behave very differently in different organisms and under different exposure conditions. In the scallops, $\mathrm{Zn}$ was redistributed among the different subcellular compartments much faster than $\mathrm{Cd}$ was, suggesting an effective regulation mechanism for $\mathrm{Zn}$.

To conclude, the study of metal toxicity has come a long way, and it is clear that significant progress has been made over the past 30 years. Collaboration between chemists and biologists is important in studying the mechanisms of metal toxicity. Metals are very different and complicated in their interactions with aquatic organisms, thus predicting their toxicity is a daunting task. To further understand metal toxicity, it is important that we continue to study the cellular fates of metals, as well as the handling and sequestration strategies of different organisms. Metal biologists should not be intimidated by simple questions such as "what is the difference between cadmium and copper" or "what is the difference between mussels and oysters". It is these differences that make metal ecotoxicology an intriguing research area. Given the frequent metal pollution incidents in China, metal ecotoxicology should certainly be a major research focus. A fundamental understanding of the environmental and biological behavior of metals is necessary before remediation can be fully implemented.

I thank guest editor Dr. Cai Yong for his invitation to contribute a review article to this special issue. This work was supported by the Research Grants Council of Hong Kong (662610 and 663009), and the Program for Changjiang Scholars and Innovative Research Team in University (IRT0941).

1 Pan K, Wang W X. Trace metal contamination in coastal and estuarine environments in China. Sci Total Environ, 2012, 421/422: 3-16

2 Cheng J T, Wang W X. Effects of heavy metals on the embryonic development and larval growth of bivalve, Ostrea platicula. J Xiamen Univ (Nat Sci), 1985, 21: 96-101

3 Morel F M M. Principles of Aquatic Chemistry. New York: John Wiley \& Sons Inc., 1983

4 Stumm W, Morgan J J. Aquatic Chemistry, Chemical Equilibria and Rates in Natural Waters, 3rd ed. New York: John Wiley \& Sons Inc., 1996

5 Langston W J, Bebianno M J, Burt G R. Metal handling strategies in mollusks. In: Langston W J, Bebianno M J, eds. Metal Metabolism in Aquatic Environments. London: Chapman \& Hall, 1998. 219-283

6 Rainbow P S. Trace metal concentrations in aquatic invertebrates: Why and so what. Environ Pollut, 2002, 120: 497-507

7 Luoma S N, Rainbow P S. Metal Contamination in Aquatic Environments: Science and Lateral Management. Cambridge: Cambridge University Press, 2008

8 Wang W X. Trace Metal Ecotoxicology and Biogeochemistry. Beijing: Science Press, 2011. 1-322

9 Wang W X. Incorporating exposure into aquatic toxicological studies: An imperative. Aquat Toxicol, 2011, 105S: 9-15

10 Wang W X, Rainbow P S. Subcellular partitioning and the prediction of Cd toxicity to aquatic organisms. Environ Chem, 2006, 3: 395-399

11 Campbell P G C. A critique of the free-ion activity model. In: Tessier A, Turner D R, eds. Metal Speciation and Bioavailability in Aquatic systems. Chichester: John Wiley \& Sons, 1995. 45-102

12 Di Toro D M, Allen H E, Bergman H L, et al. Biotic ligand model of the acute toxicity of metals. 1. Technical basis. Environ Toxicol Chem, 2001, 20: 2383-2396

13 Paquin P R, Gorsuch J W, Apte S, et al. The biotic ligand model: A historical overview. Comp Biochem Physiol, 2002, C133: 3-35

14 Campbell P G C, Errecalde O, Fortin C, et al. Metal bioavailability to phytoplankton: Applicability of the biotic ligand model. Comp Biochem Physiol, 2002, 133: 189-206

15 Slaveykova V I, Wilkinson K J. Predicting the bioavailability of metals and metal complexes: Critical review of the biotic ligand model. Environ Chem, 2005, 2: 9-24

16 Wallace W G, Lopez G R. Relationship between subcellular cadmium distribution in prey and cadmium trophic transfer to a predator. Estuaries, 1996, 19: 923-930

17 Wallace W G, Lopez G R. Bioavailability of biologically sequestered cadmium and the implications of metal detoxification. Mar Ecol Prog Ser, 1997, 147: 149-157

18 Wang D C, Couillard Y, Campbell P G C, et al. Changes in subcellular metal partitioning in the gills of freshwater bivalves (Pyganodon grandis) living along an environmental cadmium gradient. Can J Fish Aquat Sci, 1999, 56: 774-784

19 Blackmore G, Wang W X. Uptake and efflux of Cd and Zn by the green mussel Perna viridis after metal preexposure. Environ Sci Technol, 2002, 36: 989-995

20 Wallace W G, Lee B G, Luoma S N. Subcellular compartmentaliza- 
tion of $\mathrm{Cd}$ and $\mathrm{Zn}$ in two bivalves. I. Significance of metal-sensitive fractions (MSF) and biologically detoxified metal (BDM). Mar Ecol Prog Ser, 2003, 249: 183-197

21 Wallace W G, Luoma S N. Subcellular compartmentalization of Cd and $\mathrm{Zn}$ in two bivalves. II. The significance of trophically available metal (TAM). Mar Ecol Prog Ser, 2003, 257: 125-137

22 Rainbow P S, Luoma S N, Wang W X. Trophically available metal: A variable feast. Environ Pollut, 2011, 159: 2347-2349

23 Zhang L, Wang W X. Significance of subcellular metal distribution in prey in influencing the trophic transfer of metals in a marine fish. Limnol Oceanogr, 2006, 51: 2008-2017

24 Cheung M S, Wang W X. Influence of subcellular metal compartmentalization in different prey on the transfer of metals to a predatory gastropod. Mar Ecol Prog Ser, 2005, 286: 155-166

25 Miao A J, Wang W X. Cadmium toxicity to two marine phytoplankton under different nutrient conditions. Aquat Toxicol, 2006, 78: $114-126$

26 Miao A J, Wang W X. Copper accumulation, toxicity and low molecular weight thiols synthesis in different nutrient-conditioned diatoms. Environ Sci Technol, 2007, 41: 1777-1782

27 Wang M J, Wang W X. Cadmium toxicity in a marine diatom as predicted by the cellular metal sensitive fraction. Envion Sci Technol, 2008, 42: 940-946

28 Wang M J, Wang W X. Temperature-dependent sensitivity of a marine diatom to cadmium stress explained by subcellular distribution and thiol synthesis. Envion Sci Technol, 2008, 42: 8603-8608

29 Grill E, Winnacker E L, Zenk M H. Phytochelatins: The principal heavy metal complexing peptides of higher-plants. Science, 1985, 230: 674-676

30 Wang M J, Wang W X. Cadmium in three marine phytoplankton: Accumulation, subcellular fate and thiol induction. Aquat Toxicol, 2009, 95: 99-107

31 Wu Y, Wang W X. Accumulation, subcellular distribution and toxicity of inorganic mercury and methylmercury in marine phytoplankton. Environ Pollut, 2011, 159: 3097-3105

32 Wallace W G, Brouwer T M, Lopez G R. Alterations in prey capture and induction of metallothioneins in grass shrimp fed cadmium-contaminated prey. Environ Toxicol Chem, 2000, 19: 962-971
33 Perceval O, Coullard Y, Pine-Alloul B, et al. Metal-induced stress in bivalves living along a gradient of $\mathrm{Cd}$ contamination: Relating subcellular metal distribution to population-level responses. Aquat Toxicol, 2004, 69: 327-345

34 Perceval O, Coullard Y, Pine-Alloul B, et al. Linking changes in subcellular cadmium distribution to growth and mortality rates in transplanted freshwater bivalves (Pyganodon grandis). Aquat Toxicol, 2006, 79: 87-98

35 Kraemer L D, Campbell P G C, Hare L. Seasonal variations in hepatic $\mathrm{Cd}$ and $\mathrm{Cu}$ concentrations and in the sub-cellular distribution of these metals in juvenile yellow perch (Perca flavescens). Environ Pollut, 2006, 142: 313-325

36 Campbell P G C, Giguere A, Bonneris E, et al. Cadmium handling strategies in two chronically exposed indigenous freshwater organisms-the yellow perch (Perca flavescens) and the floater mollusc (Pyganodon grandis). Aquat Toxicol, 2005, 72: 83-97

37 Giguere A, Campbell P G C, Hare L, et al. Sub-cellular partitioning of cadmium, copper, nickel and zinc in indigenous yellow perch (Perca flavescens) sampled along a polymetallic gradient. Aquat Toxicol, 2006, 77: 178-189

38 Tsui M T K, Wang W X. Acute toxicity of mercury to Daphnia magna under different conditions. Environ Sci Technol, 2006, 40: 4025-4030

39 Wang W X, Guan R. Subcellular distribution of zinc in Daphnia magna and implication for toxicity. Environ Toxicol Chem, 2010, 29: 1841-1848

40 Shaw J R, Dempsey T D, Chen C Y, et al. Comparative toxicity of cadmium, zinc, and mixtures of cadmium and zinc to daphnids. Environ Toxicol Chem, 2006, 25: 182-189

41 Rainbow P S, Luoma S N. Metal toxicity, uptake and bioaccumulation in aquatic invertebrates-Modelling zinc in crustaceans. Aquat Toxicol, 2011, 105: 455-465

42 Ng T Y T, Wang W X. Dynamics of metal subcellular distribution and its relationship with metal uptake in marine mussels. Environ Toxicol Chem, 2005, 24: 2365-2372

43 Pan K, Wang W X. The subcellular fate of cadmium and zinc in the scallop Chlamys nobilis during waterborne and dietary metal exposure. Aquat Toxicol, 2006, 90: 253-260

Open Access This article is distributed under the terms of the Creative Commons Attribution License which permits any use, distribution, and reproduction in any medium, provided the original author(s) and source are credited. 